\title{
A novel approach for detection of neurological disorders through electrical potential developed in brain
}

\author{
Mohd Suhaib Kidwai, S. Hasan Saeed \\ Department of Electronics and Communication Engineering, Integral University, India
}

\begin{tabular}{|c|c|}
\hline Article Info & ABSTRACT \\
\hline Article history: & \multirow{5}{*}{$\begin{array}{l}\text { This paper talks about the phenomenon of recurrence and using this concept } \\
\text { it proposes a novel and a very simple and user friendly method to diagnose } \\
\text { the neurological disorders by using the EEG signals. The mathematical } \\
\text { concept of recurrence forms the basis for the detection of neurological } \\
\text { disorders, and the tool used is MATLAB. Using MATLAB, an algorithm is } \\
\text { designed which uses EEG signals as the input and uses the synchronizing } \\
\text { patterns of EEG signals to determine various neurological disorders through } \\
\text { graphs and recurrence plots. }\end{array}$} \\
\hline Received Sep 3, 2018 & \\
\hline Revised Jan 6, 2019 & \\
\hline Accepted Mar 11, 2019 & \\
\hline Keywords: & \\
\hline
\end{tabular}

Coupling index

EEG signals

Order recurrence plot

Recurrence

Copyright $\odot 2019$ Institute of Advanced Engineering and Science. All rights reserved.

\section{Corresponding Author:}

Mohd Suhaib Kidwai,

Department of Electronics and Communication Engineering,

Integral University,

Kursi Road, Lucknow, India.

Email: dan_kdw@rediffmail.com

\section{INTRODUCTION}

Recurrence is a fundamental characteristic of many dynamical systems. This recurrence property is exploited to characterise the system's behaviour in phase space. The concept of recurrence is used for the analysis of data and to study dynamical systems. It is a powerful tool for the visualisation of dynamical systems and analysis [1]. Human body also comprises of vital organs like brain, which produces EEG signals.

These signals are highly random and dynamical in nature and are difficult to analyse by conventional methods as the changes occur at very short durations. Since many disorders manifest themselves in the patterns and synchronization of these random signals (like EEG and ECG ssignal), the concept of recurrence can be used to diagnose and detect several disorders by analyzing the recurrence phenomenon in biosignals.

There are already the methods for diagnosis of neurological disorders ,but they are costly,disease specific i.e. a specific instrument or setup can be used to diagnose only one type of disorder,and some of them also require a sensor or a device to be implanted inside a human body for taking readings, which can be the cause of infections.Moreover, the existing diagnostic methods also requires great expertise for taking the readings and analyzing them [2-4].

This paper gives the keen insight of the process of recurrence in human body and proposes a novel method to detect the neurological disorders like epileptic seizures, Alzheimer's disease etc. The proposed method uses the concept of recurrence and tells the degree of coupling or synchronism between the EEG signals taken from various different positions from the human brain [5].

A parameter called the"synchronization Index", is used to detect the recurrence patterns in the EEG signals from various channels of EEG machine and a graph is plotted using MATLAB. It is seen that during neurological disorders the synchronism between EEG signals taken from different positions of brain 
increases. This can be visualized using the graphs. This new method of diagnosing the neurological disorders is a simple and economic technique that eliminates the limitations of the existing techniques.

The proposed algorithm of this paper can be used to diagnose many neurological disorders without any changes in setup and without putting anything inside the body for recording purpose. Moreover, it provides better understanding and quantitative analysis of the neurological disorder, with the help of graphs.

1. Significance of Detection of Coupling in Biomedical System: Natural systems are typically highly complex, and so also are the signals derived from them. This is especially true of the cardiovascular system and brain, and an enormous amount of effort has been made in recent years to develop time series analysis for diagnostic applications, i.e., to find ways to determine the physiological state by analysis of the corresponding complex signals. Synchronization and related phenomena in coupled complex systems have been found to occur, not only in physical, but also in many biological systems, e.g., the cardio respiratory interaction and neural signals. Measures of complexity have been developed that distinguish between regular, chaotic, and random behaviors, and can try to predict a heart attack or epileptic seizure. It has been reported that complexity of heart and brain data can distinguish healthy and sick subjects and sometimes even predict heart attack or epileptic seizure. The main types of complexity parameters are entropies, fractal dimensions, and Lyapunov exponents.

2. Non Linear signal processing: Depending on the nature of the systems, there are different requirements to the methods. While linear methods based on correlations are not sufficient to deal with nonlinear dependencies, most nonlinear methods require sufficiently long stationary time series. For the case that stationarity holds only for short observation time, cross recurrence plots (CRPs) were introduced. However, this method is based on taking distances of trajectories, which is conceptually difficult on physically different systems. A general problem in studying multivariate data from natural systems, for instance EEG data, is that measurement conditions change with time. Among others offset and amplitude range can vary differently within the channels. To overcome this problem we have consider a special symbolic dynamics of the system, where the time series is encoded by order patterns. This yields further symbol sequences, which are invariant with respect to certain distortions in amplitude.

\section{RECURRENCES}

The concept of recurrence goes back to Poincare, who proved that after a sufficiently long time, the trajectory of a chaotic system in phase space will return arbitrarily close to any former point of its route with probability one.

\subsection{Types of recurrences}

The types of recurrences can be explained briefly by the Table 1 .

\begin{tabular}{cccc}
\hline Recurrence Matrix & Cross Recurrence Matrix & Joint Recurrence Matrix & Order Recurrence Matrix \\
\hline & & & $\operatorname{ORP}(\mathrm{t}, \tau)=1, \pi_{\mathrm{x}}(\mathrm{t})=\pi_{\mathrm{y}}(\mathrm{t}+\tau)$ \\
0, otherwise \\
$\mathrm{R}_{\mathrm{ij}=} \Theta\left(\varepsilon-\left\|\mathrm{x}_{\mathrm{i}}-\mathrm{x}_{\mathrm{j}}\right\|\right)$ & $\mathrm{CR}_{\mathrm{ij}=} \Theta\left(\varepsilon-\left\|\mathrm{x}_{\mathrm{i}}-\mathrm{y}_{\mathrm{j}}\right\|\right)$ & $\mathrm{JR}_{\mathrm{ij}}^{\mathrm{xy}}=\mathrm{R}_{\mathrm{ij}}^{\mathrm{x}} * \mathrm{R}_{\mathrm{ij}}^{\mathrm{y}}$ & $* \begin{array}{c}\pi_{\mathrm{x}}(\mathrm{t})=1, \text { if } \mathrm{x}(\mathrm{t})>\mathrm{x}(\mathrm{t}+\tau) \\
\tau=\text { delay }\end{array}$ \\
\hline
\end{tabular}

Here,

$\varepsilon=10 \%$ of the maximum value of the signal over a given interval of time

$\mathrm{x}_{\mathrm{i}}, \mathrm{X}_{\mathrm{j}}$ are the $\mathrm{i}^{\text {th }}$ and $\mathrm{j}^{\mathrm{th}}$ sample of the same signal series and $\mathrm{R}_{\mathrm{ij}}$ is the recurrence matrix

$\mathrm{x}_{\mathrm{i}, \mathrm{y}_{\mathrm{j}}}$ are two different samples of two different signal series between which recurrence needs to found and $\mathrm{CR}_{\mathrm{ij}}$ refers to the cross recurrence

Recurrences in Bio Medical Signals: The biomedical signals like ECG and EEG are highly random in nature and vary very much even in short time duration. Superficially, it seems that there is no pattern in EEG signals, but when observed closely over the time limited samples which are obtained by digitizing and filtering one can find that, there are certain patterns of repetitions or recurrences in EEG signals taken from various positions of brain using EEG machine.

The recurrence between any two time series of biomedical signals can be found by above mentioned methods. There are various existing methods to analyze the biomedical signals by using the concept of recurrences. Some of them are as follows: 


\subsection{Decomposition model (based on coupling)}

The form of a coupling function depends on the differing contributions from individual oscillations. Changes in form may depend predominantly on only one of the phases (along one-axis), or they may depend on both phases, often resulting in a complicated and intuitively unclear dependence. This model mainly takes phase into consideration, though amplitude dynamics can also be taken into consideration. The coupling between the various signals or the recurrences is found out and disorders are diagnosed on the basis of coupling strength [3-6].

Previous coupling treatments, including the cross-frequency coupling in neuroscience, have focused on the net coupling in one direction. In this model, we decompose the net coupling into two components depending on their functional roles: the direct and the indirect couplings. Direct-coupling describes the influence of the direct (unidirectional) driving that one oscillator exerts on the other. Arguably, it is the most studied interaction in physiology, often linked to modulation mechanisms. The second component, indirectcoupling, often called common-coupling, and depends on the shared contributions of the two oscillations. The indirect coupling also includes the diffusive coupling given with the phase difference terms. The mechanism behind this coupling component or process, although we present the model in relation to phase dynamics, a similar functional decomposition of the couplings can also be applied to amplitude dynamics.

In terms of the general theory of phase dynamics and equation, the coupling function $q_{1}\left(\phi_{1}, \phi_{2}\right)$ can be expressed as the product of two functions [4-12]

$$
q_{1}\left(\phi_{1}, \phi_{2}\right)=\mathrm{P}_{1}\left(\phi_{1} \mathrm{P}_{2}\left(\phi_{2}\right)\right.
$$

where $\mathrm{P}_{1}\left(\phi_{1}\right)$ is the phase response curve (PRC) of the first oscillator and shows how it responds to external perturbations, while $\mathrm{P}_{2}\left(\phi_{2}\right)$ is the perturbation function through which the second oscillator acts on the first one.

\subsection{Signal processing and statistical analysis}

The signals were first inspected visually, followed by automated artifact removal by interpolation. Data from subjects whose signals had many artifacts were disregarded and not analyzed. The cross-frequency intervals were estimated by standard digital filtering procedures, including a FIR filter followed by a zerophase digital filtering procedure to ensure that no time or phase lags were introduced by the filtering. The boundaries of the intervals extracted from the EEG signal were $\delta=0.8-4 \mathrm{~Hz}, \theta=4-7.5 \mathrm{~Hz}, \alpha=7.5-14 \mathrm{~Hz}$, $\beta=14-22 \mathrm{~Hz}$ and $\gamma=22-100 \mathrm{~Hz}$; the interval extracted from the respiration signal was $r=0.145-0.6 \mathrm{~Hz}$; and the extraction of the heart activity from the ECG signal was $h=0.6-2 \mathrm{~Hz}$. Wavelet power and coherence analyses, together with further clinical interpretation, will be presented elsewhere. For the EEG oscillations special care was taken in dealing with frequency spillage between intervals, heart artifacts and power line artifacts. Then the coupling between these obtained ECG signals and those obtained from EEG machine is studied and compared with the standard values of various statistical parameters, which are obtained from the healthy person. Following parameters can be used to study the recurrence pattern in the biomedical signals:

a. Determinism(DET): These measures are based on the histogram $P(l)$ of diagonal lines of length $l$. Processes with uncorrelated or weakly correlated, stochastic or chaotic behavior cause none or very short diagonals, whereas deterministic processes cause longer diagonals and less single, isolated recurrence points. Therefore, the ratio of recurrence points that form diagonal structures (of minimum length $l \mathrm{~min}$ ) to all recurrence points is introduced as a measure for determinism (or predictability) of the system.

A diagonal line of length $l$ means that a segment of the trajectory is rather close during 1 time step to another segment of the trajectory at a different time; thus, these lines are related to the divergence of the trajectory segments. The average diagonal line length is the average time that two segments of the trajectory are close to each other, and can be interpreted as the mean prediction time.

b. Measures based on vertical lines: These measures are based on the histogram $P(v)$ of vertical lines of length $v$. The ratio between the recurrence points forming the vertical structures and the entire set of recurrence points is called laminarity [13].

The computation of LAM is realized for those $\mathrm{v}$ that exceed a minimal length vmin in order to decrease the influence of the tangential motion. LAM represents the occurrence of laminar states in the system without describing the length of these laminar phases. It will decrease if the RP consists of more single recurrence points than vertical structures. The average length of vertical structures is called trapping time (TT). 


\subsection{The proposed method}

The mathematical concept of recurrence is the crux of the algorithm. The EEG signals from various channels of EEG machine are taken as an input which will be used by the program in MATLAB. This program will be based on the proposed algorithm and basically compares the synchronism between the EEG signals of any two channels. This comparison is done by using the concept of recurrence. Second thing is to find the degree of recurrence which is found out by using the formula of synchronization index $\rho \pi$ [14].

The advantage of using synchronization index in this algorithm is that it serves two purposes, firstly it helps in understanding the synchronization between the two signals from EEG channels and secondly it allows the user to find the degree of synchronization. Using the plot command of MATLAB, this synchronization index can be shown in form of graphs .If the graph shows increasing pattern, it shows high amount of synchronization between the two signals else the low synchronization. Following is the diagrammatic representation of the proposed algorithm for better visualization and understanding [15-27]

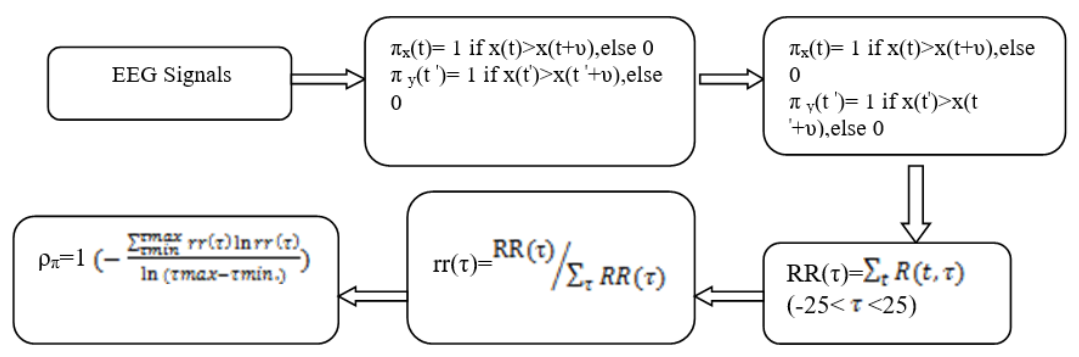

Figure 1. The block diagram of the proposed algorithm

The steps of algorithm, whose block diagram is depicted as Figure 1, can be defined as follows:

a. The signals from the channels of EEG machines are taken and put in a form of array so that it can be used as an input.

b. The signals from the channels of EEG machines are taken and put in a form of array so that it can be used as an input in MATLAB program based on this algorithm.

c. pie $\mathrm{x}(\mathrm{)}$ refers to the order pattern and it is found out by comparing samples by their respective values at different intervals. Similarly pie y $\left(\pi_{\mathrm{y}}\right)$ is found.

d. Once we have the values of pie $\mathrm{x}$ and pie $\mathrm{y}$ in form of matrices next step is to find order pattern. It is found as follows:

$\operatorname{ORP}(\mathrm{t}, \tau)=1$ if $\pi_{\mathrm{x}}(\mathrm{t})=\pi_{\mathrm{y}}(\mathrm{t}+\tau)$

0 , otherwise

Next step is to find out the recurrence rate and its normalized value. This is done using the following formulas:

$$
\begin{aligned}
& \operatorname{RR}(\tau)=\sum_{\mathrm{t}} \mathrm{R}(\mathrm{t}, \tau) \\
& \operatorname{rr}(\tau)=\operatorname{RR}(\tau) / \sum_{\tau} \operatorname{RR}(\tau)
\end{aligned}
$$

Then finally a parameter called "synchronization index" $\left(\rho_{\pi}\right)$ is found and plotted. The formula for synchronization index is as follows:

$$
\rho_{\pi}=1-\left(-\frac{\sum_{\tau \min }^{\tau \max } r(\tau) \ln r r(\tau)}{\ln (\tau \max -\tau \min .)}\right)
$$

This parameter " $\rho_{\pi}$ ", when plotted between its two channels using MATLAB, will show either high, moderate, low or no synchronization between the signals taken from different channels of EEG. Based on the value of synchronization index, one can compare the synchronism between EEG signals obtained from the different channels from the subject's brain. Moreover the recurrence plots are also plotted which helps in better understanding of synchronism between the EEG [28-30]. 


\section{RESULTS AND ANALYSIS}

Through the proposed algorithm, certain programs have been made which takes EEG signals of patient (from different channels of same EEG machines), as an input. Their has been a common observation in most of the neurological diseases that at certain point of time,the EEG signals show high degree of coupling or synchronization. This feature is ascertained with the help of recurrence plot and the plot of synchronization/coupling index to detect the disorders. The results for some of the neurological disease are as follows:

a. Epileptic seizure: Using the recurrence plot and the coupling index,one can easily detect the epileptic seizure and its intensity.The ORP and the coupling index graph of a person with epilepsy is shown under: Two EEG signals are taken from different channels as shown in Figures 2 and 3. Figure 4 shows that there are RP of EEG at different moments. It is found that density of black dots is diminishing as we move towards seizure moments and eventually RP has very rare white black dots at the seizure activity in Figures 5 and 6 . This is also shown through recurrence rate $\mathrm{rr}(\mathrm{t})$ and coupling index $\rho \pi$ in next figures.

The increasing trend in the coupling index in Figure 5 shows high degree of coupling or synchronization between EEG signals taken from channels. This happens when the person is suffering from epileptic fits. This is the way to diagnose epilepsy using the proposed method.

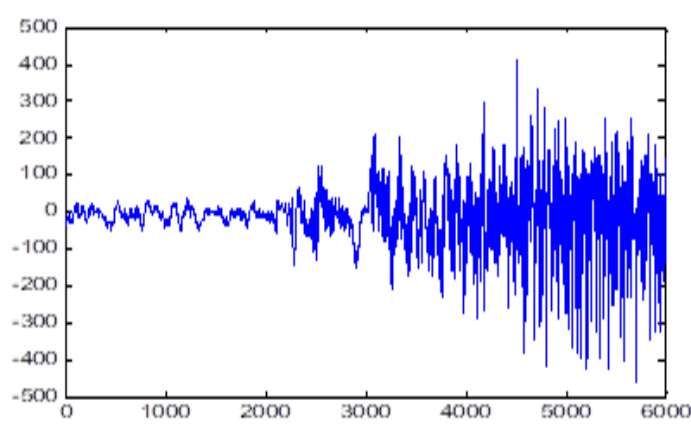

Figure 2. EEG signal of a patient with Epilepsy from Channel 1 of EEG machine

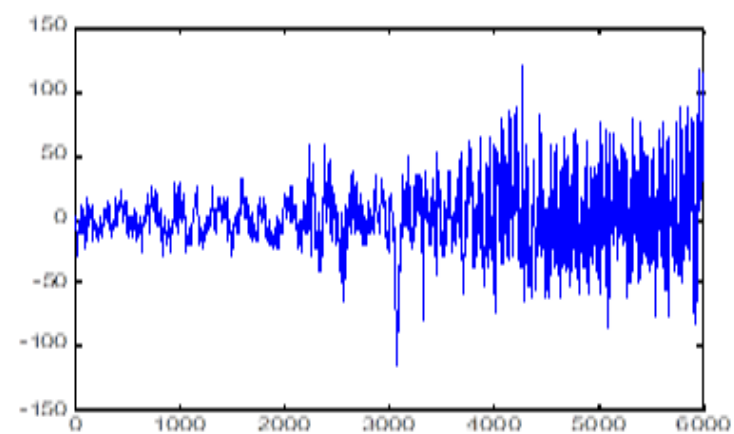

Figure 3. EEG signal of a patient with Epilepsy from Channel 17 of EEG machine
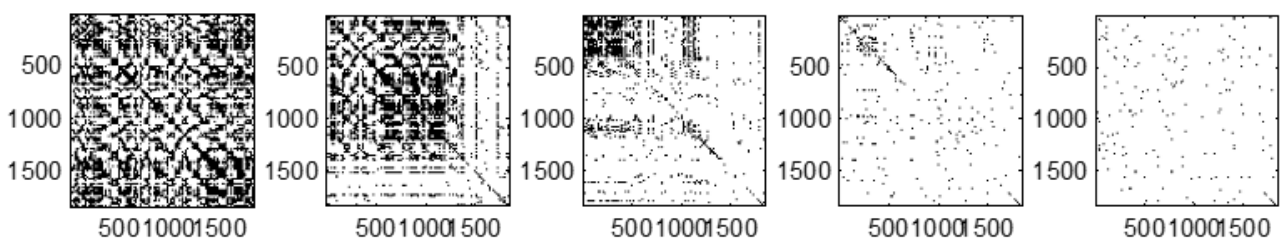

Figure 4. RP of EEG at different moments

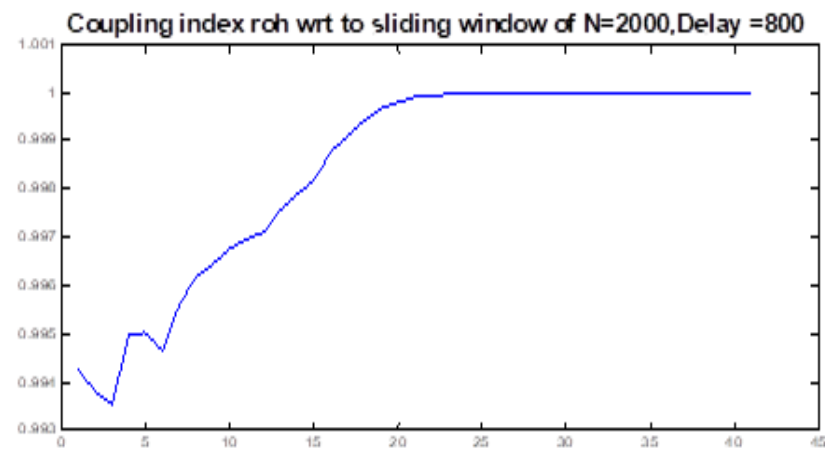

Figure 5. Synchronization index graph of above patient with epilepsy 
Figures 6-8 shows the graph of synchronization index. The channels are the channels of EEG machine. EEG signals from different parts of brain are recorded using the EEG machine and then the two channels are taken at a time and graph is plotted. All the graphs shows the increasing trend in synchronization index graph and this shows that the person is having epilepsy.This instant where the graph of synchronization index has started increasing, shows the onset of epileptic seizure.

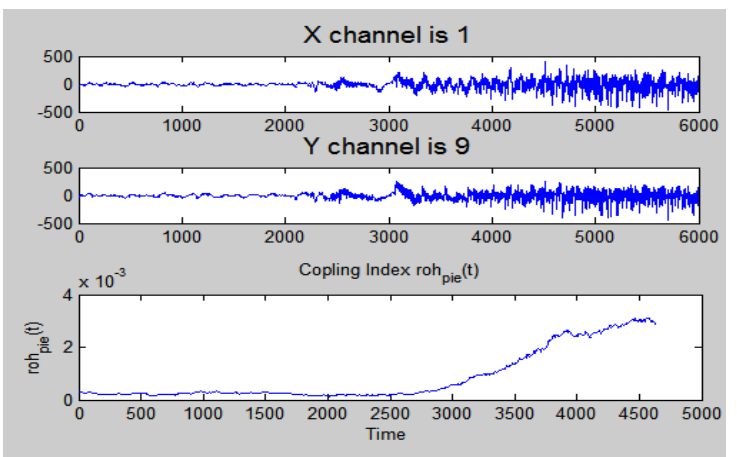

Figure 6. Coupling index graph and EEG signals of channel 1 and 9

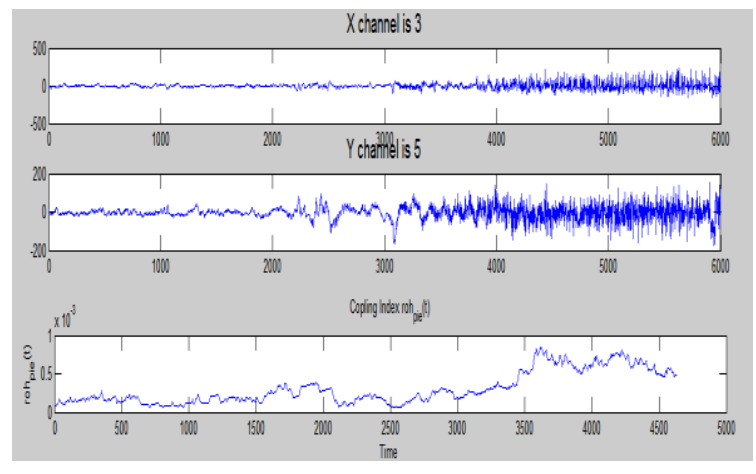

Figure 7. Coupling index graph and EEG signals of channel 3 and 5

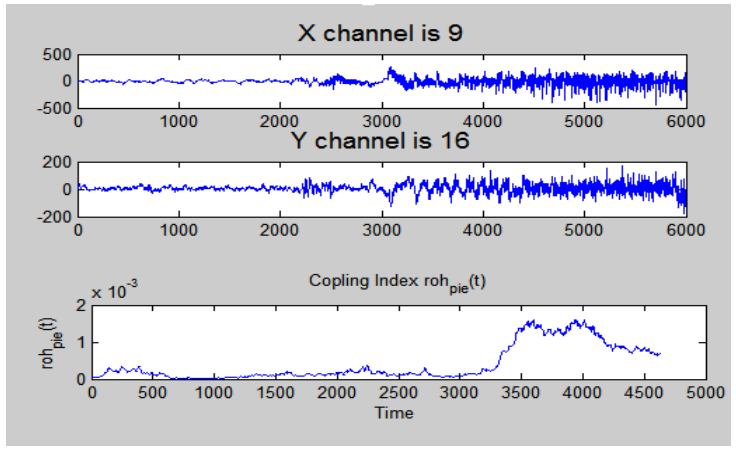

Figure 8. Coupling index graph and EEG signals of channel 9 and 16

b. Bruxism: This is an involuntary activity which involves excessive grinding or clenching of teeth. This is also a neurological disorder and during this phenomenon, there is a high degree of phase locking or synchronization, hence the proposed algorithm can also be used to diagnose this disorder. In this process the EEG signals of the subject are taken routinely for at least a week and then using this algorithm, the synchronization index is plotted. The increasing trend in synchronization index ensures that the most probable reason out of the various reasons for teeth grinding is bruxism. The result of a patient, which is obtained by using this algorithm is given Figure 9.

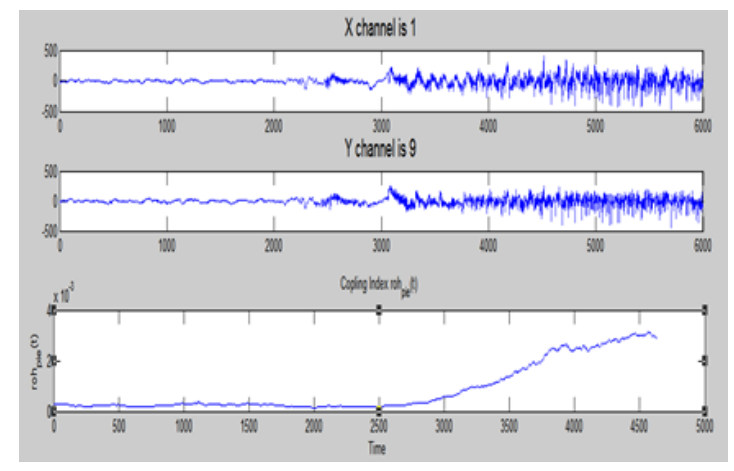

Figure 9. Coupling index graph and EEG signals of channel 1 and 9 
Using the proposed algorithm, the synchronization index graph is plotted between different channels. The increasing trend shows that there is a presence of high synchronization between EEG signals at certain interval of time. This, is case of bruxism shows the time when the subject under observation has started clenching the teeth involuntarily.

c. Alzheimer's disease: This is also a neurological disorder, which manifests itself in the phase locking or increased coupling between the EEG signals obtained from various regions of a patient's brain. This disease is also characterized by the repetitive patterns in EEG signals that are taken from various channels of EEG machine.Therefore this proposed method can also be used to diagnose the Alzheimer's disease by plotting the graph of synchronization index between the EEG signals obtained from different regions of brain. The high values of synchronization or the rising pattern of the curve proves that the patient has the traits of Alzheimer's disease. Figures 10 and 11 is the result of the Alzheimer patient obtained by the proposed method. Two channels are taken at a time and the graph is plotted between them. The rising pattern of synchronization index between various channels shows that the person is suffering from Alzheimer disease. Likewise, other neurological diseases like autism, dementia etc, that manifest themselves in coupling of EEG signals can effectively be diagnosed using the proposed method.

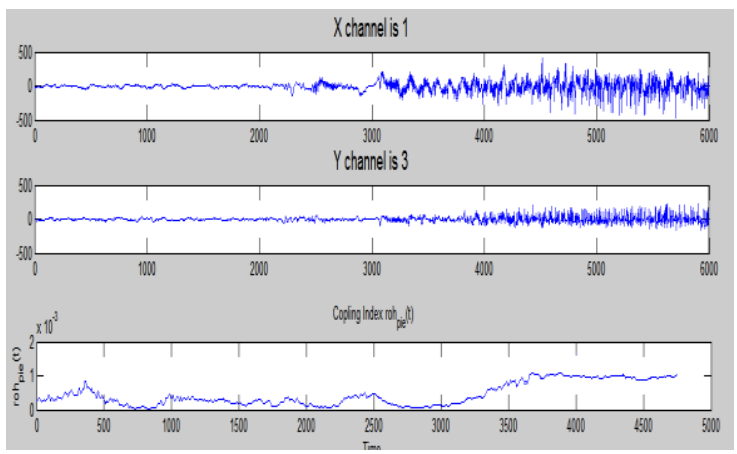

Figure 10. Coupling index graph and EEG signals of channel 1 and 3

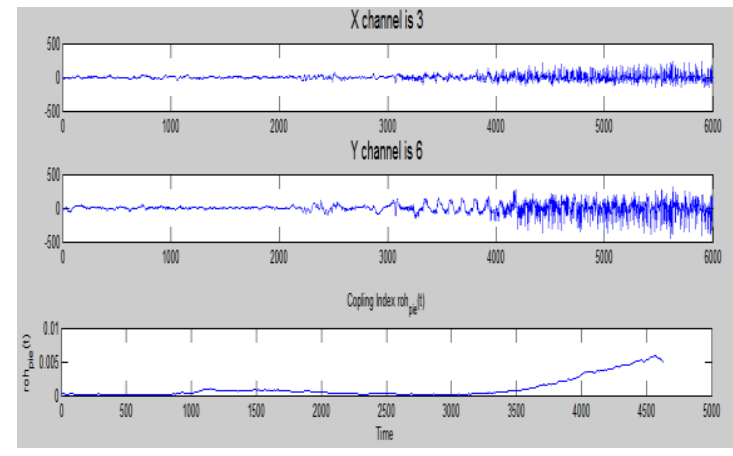

Figure 11. Coupling index graph and EEG signals of channel 3 and 6

\section{DISCUSSION}

Comparison with the Decomposition Model: Decomposition model mainly focuses on finding the synchronism between the two or more signals using the phase , as a parameter. Now the problem with this method is that phase decomposition techniques are complex and often require associated circuitry which increases the overall cost of the device or the machine which will use the algorithm based on this model.Contrary to this, the algorithhem proposed in this paper,will find the synchronism between the two signals by using the simple mathematical concept of recurrences. The proposed algorithm simply uses the generalized formula of "Synchronization $\operatorname{Index}(\rho \pi)$ ". This approach ensures that no additional circuitry is involved as there is no need to extract any phase related information from the signal.So this method is cost effective and simple.

Comparison with model based on signal processing and statistical analysis: The various approaches which use signal processing methods generally use the algorithms like KNN algorithm etc. These algorithms are very application specific,so suppose if they are designed for a specific application, like for detecting epileptic seizure, then if that same method is used for finding say, Alzheimer, then lot of changes needs to be done in source codeand source program.But if we use thesynchronization index as a parameter to find the neurological disorder,it will serve as a uniform and generalized method,which can be used to detect any neurological disorder that manifest itself in the synchronization of signals. Similarly, if we compare the proposed algorithm with methods based on statistical analysis, we will see that methods based on statistical analysis use various parameters like determinism, laminarity etc to determine synchronism, while the proposed algorithm only uses a single parameter "synchronization Index", so obviously the latter approach is simpler as compared to the former one. 


\section{CONCLUSION}

To conclude, this method altogether presents a new approach for detection of neurological diseases that have certain degree of coupling or phase locking between them. Since the concept of order patterns is used which only compares the discrete values and creates a matrix of one and zero, the error probability in this method is very low. Secondly, the algorithm used in this method is robust and not disease specific and it can be use to detect any neurological disorder that manifest itself in EEG signals. Moreover, this method is a non invasive method so the chances of any kind of infection is nil. This method also paves the way for developing the more efficient algorithms which can also use ECG and EMG signals to diagnose various disorders.

\section{REFERENCES}

[1] A. Bahraminasab, et al., "Direction of Coupling from Phases of Interacting Oscillators: A Permutation Information Approach," Physical Review Letters, vol. 100, 2008.

[2] A. Groth, "Visualization of coupling in time series by order recurrence plots," Physical Review, vol. 72, 2005.

[3] A. Pikovsky, et al., "Synchronization- A Universal Concept in Nonlinear Sciences," Cambridge University Press, Cambridge, England, 2001.

[4] A. Stefanovska, et al., "Reversible Transitions between Synchronization States of the Cardiorespiratory system." Phys Rev Lett. Nov 27;85(22):4831-4, 2000.

[5] Sacred Heart Health Care System, "Ambulatory Electroencephalography (EEG) Cardiorespiratory System" Phys. Rev. Lett., vol. 85, pp. 4831, 2000.

[6] M. Niknazar, et al., "A New Framework Based on Recurrence Quantification Analysis for Epileptic Seizure Detection," IEEE Journal of Biomedical and Health Informatics, vol/issue: 17(3), 2013.

[7] J. Engel, et al., "Epilepsy: A Comprehensive Textbook," Lippincott Williams \& Wilkins, 2007.

[8] B. Litt, et al., "Translating Seizure Detection, Prediction and Brain Stimulation Into Implantable Devices for Epilepsy," in Conference Proceedings in the 1st International IEEE EMBS Conference on Neural Engineering, pp. 485-488, 2003.

[9] B. Litt and K. Lehnertz, "Seizure Prediction and the Preseizure Period," Current Opinion in Neurology, vol/issue: 15(2), pp. 173-177, 2002.

[10] S. Sanei and J. Chambers, "EEG Signal Processing," Wiley-Interscience, 2007.

[11] B. Schelter, et al., "Do FalsePredictions of Seizures Depend on the State of Vigilance?" Epilepsia, vol/issue: 47(12), pp. 2058-2070, 2006.

[12] R. Schuyler, et al., "EpilepticSeizure Detection," Engineering in Medicine and Biology Magazine, IEEE, vol/issue: 26(2), pp. 74-81, 2007.

[13] B. Shneker and N. Fountain, "Epilepsy,” Disease-a-Month, vol/issue: 49(7), pp. 426-478, 2003.

[14] M. Winterhalder, et al., "The seizure prediction characteristic: a general framework to assess mand compare seizure prediction methods," Epilepsy and Behavior, 2003.

[15] M. H. D. Mohammadi, "Improved Denoising Method for Ultrasonic Echo with Mother Wavelet Optimization and best basis selection," International Journal of Electrical and Computer Engineering (IJECE), vol/issue: 6(6), 2016.

[16] N. Mohan, "Modified Variational Mode Decomposition for Power Line Interference Removal in ECG Signals," International Journal of Electrical and Computer Engineering (IJECE), vol/issue: 6(1), 2016.

[17] Mohd Suhaib Kidwai, "A Review of the Recurrence Based Techniques for Detection of Various Neurological Disorders," IJARCCE, Vol 5 Issue 9, 2016.

[18] Mohd Suhaib Kidwai, "A Novel Approach to Study the Effects of Anesthesia on Respiratory Signals by using the EEG Signals”, International Journal of Electrical and Computer Engineering (IJECE), Vol 7 Issue 6, 2017.

[19] Mohd Suhaib Kidwai, "Use Of Recurrence For Detection of Epilepsy," IJAIEM, Vol 2, Issue 5,2013

[20] Mohd Suhaib Kidwai," An Efficient Algorithm for the Diagnosis of Alzheimer Disease by Using MATLAB", IJETTCS, Vol 3 Issue 2, 2014.

[21] M. Thiel, M. C. Romano, J. Kurths, R. Meucci, E. Allaria, T. Arecchi, "Influence of observational noise on the recurrence quantification analysis," Physica D 171(3), 138, 2002.

[22] M. Thiel, M. C. Romano and J. Kurths, "Analytical Description of Recurrence Plots of White Noise and Chaotic Processes," Applied Nonlinear Dynamics 11(3), 20, 2003.

[23] M. Thiel, M. C. Romano, P. Read, and J. Kurths, "Surrogate based Hypothesis Test without Surrogates," Chaos 14 (2), 234, 2004.

[24] N. F. Rulkov, M.M. Sushchik, L. S. Tsimring, and H. D. I. Abarbanel, "Generalized Synchronization of Chaos in Directionally Coupled Chaotic System," Phys. Rev. E 51, 980, 1995.

[25] P. Faure, H. Korn, "A new method to estimate the Kolmogorov entropy from recurrence plots: its application to neuronal signals," Physica D 122, 265, 1998

[26] P. Grassberger and I. Procaccia, "Estimation of the Kolmogorov entropy from a chaotic signal," Phys. Rev. A 28, 2591, 1983.

[27] P. Tass, M. G. Rosenblum, J. Weule, J. Kurths, A. Pikovsky, J. Volkmann, A. Schnitzler, and H.J.Freund, "Detection of n:m Phase Locking from Noisy Data: Application to Magnetoencephalography," Phys. Rev. Lett. Vol. 81, No. 15, 1998. 
[28] R. Quian Quiroga, J. Arnhold and P. Grassberger, "Learning driver-response relationships from synchronization patterns," Phys. Rev. E 61, 5142, 2000.

[29] S. J. Schiff, P. So, T. Chang, R. E. Burke, and T. Sauer, "Detecting dynamical interdependence and generalized synchrony through mutual prediction in a neural ensemble," Phys. Rev. E 54, 6708, 1996.

[30] T. Schreiber, "Measuring Information Transfer," Phys. Rev. Lett. 85, 46, 2000.

\section{BIOGRAPHIES OF AUTHORS}

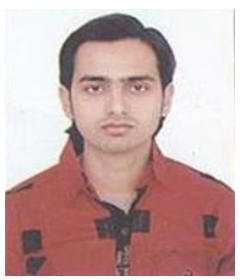

Mohd Suhaib Kidwai, is a research scholar, in the Department of Electronics and Communication, Integral University. He has interest in Digital Electronics, Signal Processing and Biomedical Electronics.

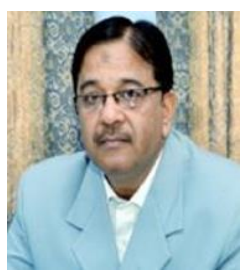

S.Hasan Saeed is currently working as Professor \& Head of the Department in Electronics \& Communication Engineering department, Faculty of Engineering at Integral University. He did his B.Tech in Electrical Engineering from Zakir HussainCollege of Engineering and Technology, campus college of Aligarh Muslim University (AMU), Aligarh, Uttar Pradesh in year 1992 and received PhD from Integral University, Lucknow in year 2010. 